\title{
The Leipzig Thyrotropin Receptor Mutation Database: Update 2012
}

\author{
J. Lüblinghoff I.T. Nebel S. Huth H. Jäschke J.Schaarschmidt M. Eszlinger R. Paschke \\ Department of Endocrinology and Nephrology, Leipzig University, Leipzig, Germany
}

Dear Sir,

Familial nonautoimmune autosomal dominant hyperthyroidism (FNAH) and sporadic congenital nonautoimmune hyperthyroidism (SNAH) are rare forms of hyperthyroidism caused by germline mutations in the TSH receptor (TSHR) gene. FNAH and SNAH are caused by constitutively activating TSHR germline mutations [1-5]. Furthermore, somatic constitutively activating TSHR mutations are found in $60-70 \%$ of single hot thyroid nodules $[6,7]$, in hot nodules of toxic multinodular goiters $[8,9]$, in hot thyroid carcinomas [10] and in small hot areas of euthyroid goiters detected by autoradiography [11]. Moreover, euthyroid hyperthyrotropinemia or primary (thyroidal) congenital hypothyroidism may be caused by inactivating (loss of function) TSHR germline mutations $[2,12]$.

In 1999 we have launched the TSH receptor mutation database compiling all reported TSH receptor mutations with their associated clinical conditions and functional characterizations [13]. An update of the database with extended functionalities such as pedigrees, search tool, or detailed functional characteristics was done in 2003 [14] and the TSHR database was installed as one of the locus-specific HUGO mutation databases (ariel.ucs.unimelb. edu.au/-cotton/glsdbq.htm). This 2003 database version contained all naturally occurring TSHR mutations reported up to December 2002 [43 activating mutations (29 somatic and 19 germline mutations) and 22 inactivating germline mutations]. Since its last revision, the TSHR database received $>25,000$ hits from $>90$ countries until December 2011. Moreover, many more TSH receptor mutations were reported during the past 10 years.

Therefore, 10 years after the last revision it is time for a further update. The updated 2012 TSHR database now contains all naturally occurring TSHR mutations reported up to April 2012. Thus the following were added:

- 5 new constitutively activating TSH receptor germline mutations causing 5 new cases of SNAH and 2 further cases with known constitutively activating TSH receptor mutations.

- 9 new constitutively activating TSH receptor germline mutations causing 10 new cases with new FNAH and 6 further families with FNAH caused by known constitutively activating TSH receptor mutations.

- 22 new constitutively activating somatic TSH receptor mutations (21 point mutations and 2 deletions) detected in 297 new hot nodules.

- 1 new somatic constitutively activating $\mathrm{TSH}$ receptor mutation causing a hot thyroid carcinoma.
- 41 inactivating TSH receptor germline mutations ( 31 point mutations, 3 deletions and 7 frameshift mutations) causing $\mathrm{TSH}$ resistance or congenital hypothyroidism.

To date 28 families with FNAH [4, 9, 15-40] and 16 cases with SNAH have been published [5, 41-55]. Moreover, up to date 105 families with euthyroid hyperthyrotropinemia or primary (thyroidal) congenital hypothyroidism have been reported [56-91].

The present TSHR database revision also includes the results of several reanalyses of previously reported constitutive activities of several TSH receptor mutations. Large variations for the extent of constitutive activity for several mutations characterized in different laboratories [92-94] and several discrepant reports have highlighted the difficulties to determine whether a TSHR mutation is constitutively active or not especially for borderline cases with only a slight increase of the basal cAMP activity. Current methods to precisely classify such mutants as constitutively active or not are limited. For characterization of constitutive TSHR in vitro activity a particular point to consider is that basal receptor activity tightly correlates with the receptor number expressed on the cell surface. Therefore, a comparison of the receptors basal activity in relation to the

\section{KARGER}

Fax +4161306 1234 E-Mail karger@karger.ch www.karger.com www.karger.com/etj
Prof. Dr. med. R. Paschke

Department of Endocrinology and Nephrology, Leipzig University

Liebigstrasse 20

DE-04103 Leipzig (Germany)

E-Mail ralf.paschke@medizin.uni-leipzig.de 
wild type is only possible with determination of the receptor cell surface expression. Three methods for the determination of constitutive TSHR activity have been described: (1) the evaluation of constitutive TSHR activity under conditions of equal receptor expression; (2) computation of the specific constitutive activity, and (3) the linear regression analysis. To date, in our view linear regression analysis is the most reproducible experimental approach to control for variable receptor expression and thus to characterize the mutant's basal in vitro activity as a function of TSHR cell surface expression. This approach utilizes a parallel measurement of basal cAMP values and receptor cell surface expression and therefore provides a reproducible decision with respect to the presence or absence of constitutive in vitro activity.

Especially if the constitutive in vitro activity is only slightly increased, the functional in vitro characterization of all new TSHR mutations should in our view be performed by linear regression analysis of constitutive activity as a function of TSHR expression [92]. Previously two TSHR germline mutations and four somatic TSHR germline mutations all previously published as constitutively active did not reveal constitutive in vitro activity in COS7 cells when later analyzed by the linear regression approach to determine constitutive activity as a function of TSHR expression $[93,94]$. Furthermore in spite of the efforts to control experimental conditions to make them more reproducible our current limitations to determine constitutive activity in vitro are illustrated by one mutation for which slight constitutive in vitro activity is only detectable with pcDNA constructs in $\mathrm{HEK}_{\mathrm{GT}}$ cells. N670S has been described in two families with FNAH [39, 40]. However, it shows lack of constitutive in vitro activity in COS-7 cells, but a detectable low constitutive in vitro activity in pcDNA constructs in $\mathrm{HEK}_{\mathrm{GT}}$ cells [94]. Moreover, silent TSHR germline mutations are known [95]. Furthermore, members of the same family harboring the same TSHR germline mutation show large differences in disease onset $[15,17,48,96]$. Therefore, besides the constitutive activation of the cAMP pathway which is modified by receptor expression and the promoter of the respective expression vector, most likely additional factors, for example genetic background, and/or iodine intake may modify the phenotypic expression [97].

The updated 2012 TSHR database is accessible under the new address http://tshreceptor-mutation-database.org with $\mathrm{R}$. Paschke as the curator. Further updates will be performed at 5 -year intervals. The references cited in this letter are available in electronic form (online suppl. references; for all online suppl. material, see www. karger.com/doi/10.1159/000342918).

\section{Disclosure Statement}

The authors declare that no financial or other conflict of interest exists in relation to the content of this article. 